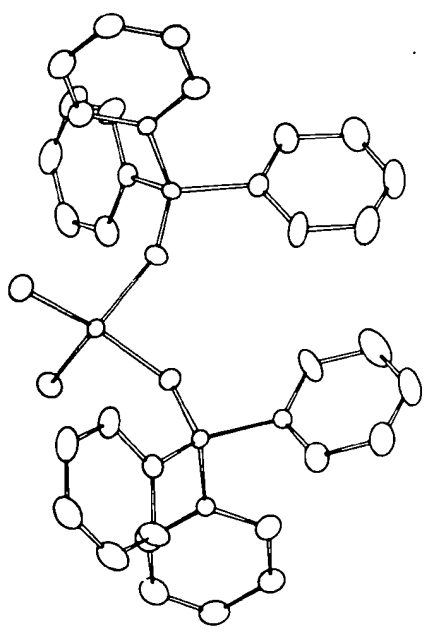

Fig. 2. View of the molecule.
This work was supported by FAPESP, CNPq and FINEP. We thank the Instituto de Física e Química de São Carlos, USP, Brazil, for computing facilities.

\section{References}

Davolos, M. R. (1978). Dissertation, Univ. of São Paulo, Brazil. Goodgame, D. M. L. \& Cotton, F. A. (1961). J. Chem. Soc. pp. 3735-3741.

Hamilton, W. C. (1959). Acta Cryst. 12, 609-610.

International Tables for X-ray Crystallography (1974). Vol. IV. Birmingham: Kynoch Press. (Present distributor D. Reidel, Dordrecht.)

JoHNSON, C. K. (1965). ORTEP. Report ORNL-3794. Oak Ridge National Laboratory, Tennessee.

Main, P., Hull, S. E., Lessinger, L., Germain, G., DeclercQ, J.-P. \& WoOlfson, M. M. (1978). MULTAN78. A System of Computer Programs for the Automatic Solution of Crystal Structures from X-ray Diffraction Data. Univs. of York, England, and Louvain, Belgium.

SHELDRICK, G. M. (1976). SHELX76. A program for crystal structure determination. Univ. of Cambridge, England.

Acta Cryst. (1985). C41, 1833-1834

\title{
Structure of a Bicyclic Sulfur-Nitrogen-Carbon Heterocyclic Molecule
}

\author{
BY A. W. CORDES \\ Department of Chemistry, University of Arkansas, Fayetteville, AR 72701, USA \\ AND R. T. OAKLEY AND RENÉ T. BOERÉ \\ Guelph-Waterloo Centre for Graduate Work in Chemistry, Guelph Campus, Department of Chemistry and \\ Biochemistry, University of Guelph, Guelph, Ontario, Canada N1G $2 W 1$
}

(Received 26 June 1985; accepted 30 July 1985)

\begin{abstract}
Phenyl- $1 \lambda^{4}, 3 \lambda^{4} \delta^{2}, 5 \lambda^{4}$-trithia-2,4,6,8,9-pentaazabicyclo[3.3.1]nonane, $\quad \mathrm{C}_{7} \mathrm{H}_{5} \mathrm{~N}_{5} \mathrm{~S}_{3}, \quad M_{r}=255 \cdot 3$, monoclinic, $P 2_{1} / n, a=5.958(1), b=22.954$ (2), $c=$ 7.428 (1) $\AA, \beta=106.25(1)^{\circ}, V=975.2(4) \AA^{3}, Z=4$, $D_{x}=1.74 \mathrm{~g} \mathrm{~cm}^{-3}, \quad \lambda($ Mo $K \alpha)=0.71073 \AA, \quad \mu=$ $7.00 \mathrm{~cm}^{-1}, F(000)=520, T=293 \mathrm{~K}, R=0.030$ for 1363 unique observed reflections. The planar SNCNS and SNSNS components of the bicyclic molecule make dihedral angles of 137.1 and $118.3^{\circ}$ with the SNS bridging unit. The $\mathrm{S}-\mathrm{N}$ bonds connecting the NSN fragment to the $\mathrm{CN}_{3} \mathrm{~S}_{2}$ ring are much longer $(1.728 \AA)$ than those in the remainder of the molecule $(1.546-$ $1.630 \AA \AA)$.
\end{abstract}

Experimental. Compound prepared by reaction of $N, N, N^{\prime}$-tris(trimethylsilyl)benzamidine with $\mathrm{S}_{3} \mathrm{~N}_{3} \mathrm{Cl}_{3}$. Crystals obtained from acetonitriie solutions. Yellow

$0108-2701 / 85 / 121833-02 \$ 01.50$ needle data crystal $0.60 \times 0.16 \times 0.14 \mathrm{~mm}$ mounted on a glass fiber. Density not measured. Intensities measured with an Enraf-Nonius CAD-4 diffractometer using variable-speed $\omega-2 \theta$ scans. Unit cell determined from least-squares analysis of angle data for 25 reflections with $20<2 \theta<28^{\circ}$. Analytical absorption correction based on crystal shape varied from 0.95 to 1.00. Data collected to $\sin \theta / \lambda$ of $0.59 \AA^{-1},-7 \leq h \leq 7$, $0 \leq k \leq 27,0 \leq l \leq 8$. Three standard reflections (252, $1,12,0, \overline{3} 73)$ varied $\pm 4.1 \%$ over $16.9 \mathrm{~h}$ of data collection; anisotropic-drift correction applied. 1839 reflections measured, 1708 unique $\left(R_{\text {int }}=0.01\right)$, 345 reflections with $I<3 \sigma(I)$ considered unobserved. Solved by direct methods using MULTAN11/82 (Main, Fiske, Hull, Lessinger, Germain, Declercq \& Woolfson, 1982) and Fourier methods. Full-matrix least squares minimized $\sum w \Delta F^{2} . \mathrm{H}$ atoms refined isotropically, other (C) 1985 International Union of Crystallography 
Table 1. Fractional atomic coordinates, isotropic thermal parameters, and their e.s.d.'s

$$
B_{\mathrm{eq}}=\frac{4}{3}\left(a^{2} B_{11}+b^{2} B_{22}+c^{2} B_{33}+a c B_{13} \cos \beta\right) \text {. }
$$

$\begin{array}{lcll}x & y & z & B_{\mathrm{eq}} / B\left(\AA^{2}\right) \\ 0.8959(1) & 0.05740(3) & 0.67029(8) & 2.57(1) \\ 0.4814(1) & 0.08893(3) & 0.72098(9) & 2.60(1) \\ 0.6221(1) & -0.02880(3) & 0.78955(9) & 2.86(1) \\ 1.0132(4) & 0.0940(1) & 0.8598(3) & 2.67(5) \\ 0.6415(4) & 0.1160(1) & 0.9165(3) & 2.98(5) \\ 0.6345(4) & 0.0824(1) & 0.5714(3) & 2.80(5) \\ 0.8576(4) & -0.0106(1) & 0.7568(3) & 3.20(5) \\ 0.4353(4) & 0.0185(1) & 0.7842(3) & 3.00(5) \\ 0.8736(4) & 0.1177(1) & 0.9533(3) & 2.38(5) \\ 0.9938(4) & 0.1513(1) & 1.1240(3) & 2.32(5) \\ 0.8903(5) & 0.1568(1) & 1.2681(4) & 2.89(6) \\ 0.9998(6) & 0.1880(1) & 1.4279(4) & 3.63(7) \\ 1.2119(6) & 0.2145(1) & 1.4424(5) & 4.00(7) \\ 1.3156(5) & 0.2092(1) & 1.3000(4) & 3.90(7) \\ 1.2099(5) & 0.1774(1) & 1.1401(4) & 3.00(6) \\ 0.743(4) & 0.141(1) & 1.256(3) & 2.5(5) \\ 0.924(4) & 0.191(1) & 1.526(3) & 2.6(6) \\ 1.278(6) & 0.234(1) & 1.548(5) & 5.3(8) \\ 1.459(5) & 0.233(1) & 1.308(4) & 4.1(7) \\ 1.275(5) & 0.177(1) & 1.036(4) & 3.5(6)\end{array}$

Table 2. Selected bond distances $(\AA)$, bond angles $\left(^{\circ}\right)$, and their e.s.d.'s

$\begin{array}{llll}\mathrm{S}(1)-\mathrm{N}(3) & 1.629(2) & \mathrm{N}(3)-\mathrm{S}(1)-\mathrm{N}(4) & 106.1(1) \\ \mathrm{S}(1)-\mathrm{N}(4) & 1.728(2) & \mathrm{N}(4)-\mathrm{S}(1)-\mathrm{N}(1) & 102.5(1) \\ \mathrm{S}(1)-\mathrm{N}(1) & 1.621(2) & \mathrm{N}(3)-\mathrm{S}(1)-\mathrm{N}(1) & 109.5(1) \\ \mathrm{S}(2)-\mathrm{N}(3) & 1.630(2) & \mathrm{N}(3)-\mathrm{S}(2)-\mathrm{N}(5) & 105.1(1) \\ \mathrm{S}(2)-\mathrm{N}(5) & 1.728(2) & \mathrm{N}(3)-\mathrm{S}(2)-\mathrm{N}(2) & 110.2(1) \\ \mathrm{S}(2)-\mathrm{N}(2) & 1.621(2) & \mathrm{N}(5)-\mathrm{S}(2)-\mathrm{N}(2) & 102.3(1) \\ \mathrm{S}(3)-\mathrm{N}(5) & 1.546(2) & \mathrm{N}(5)-\mathrm{S}(3)-\mathrm{N}(4) & 119.0(1) \\ \mathrm{S}(3)-\mathrm{N}(4) & 1.547(2) & \mathrm{S}(1)-\mathrm{N}(3)-\mathrm{S}(2) & 111.8(1) \\ \mathrm{N}(1)-\mathrm{C}(1) & 1.339(3) & \mathrm{S}(2)-\mathrm{N}(5)-\mathrm{S}(3) & 119.7(1) \\ \mathrm{N}(2)-\mathrm{C}(1) & 1.333(3) & \mathrm{S}(1)-\mathrm{N}(4)-\mathrm{S}(3) & 121.5(1) \\ \mathrm{C}(1)-\mathrm{C}(2) & 1.485(3) & \mathrm{S}(1)-\mathrm{N}(1)-\mathrm{C}(1) & 118.8(2) \\ \mathrm{C}-\mathrm{C}(\text { phenyl) range } & 1.372-1.394 & \mathrm{~S}(2)-\mathrm{N}(2)-\mathrm{C}(1) & 120.8(2) \\ \mathrm{C}-\mathrm{C}(\text { phenyl) mean } & 1.383 & \mathrm{~N}(1)-\mathrm{C}(1)-\mathrm{N}(2) & 129.8(2) \\ \mathrm{C}-\mathrm{H} \text { range } & 0.90-1.00 & \mathrm{~N}(1)-\mathrm{C}(1)-\mathrm{C}(2) & 115.6(2) \\ \mathrm{C}-\mathrm{H} \text { mean } & 0.95 & \mathrm{~N}(2)-\mathrm{C}(1)-\mathrm{C}(2) & 114.6(2) \\ & & \mathrm{C}-\mathrm{C}-\mathrm{C} \text { range } & 119.1-120.9 \\ & & \mathrm{C}-\mathrm{C}-\mathrm{H} \text { range } & 117-123\end{array}$

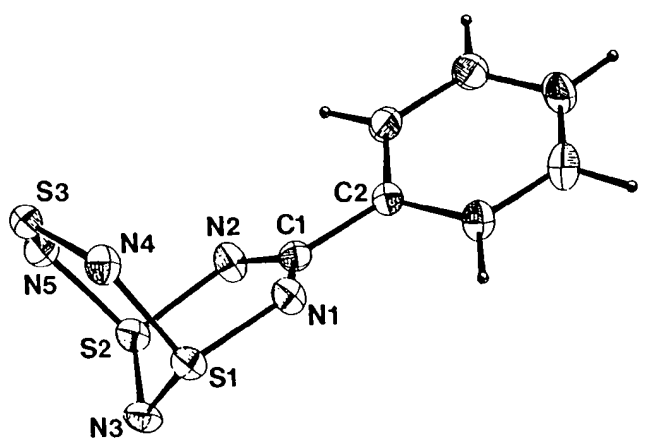

Fig. 1. ORTEPII diagram (Johnson, 1976) and atom-numbering scheme. Non-H ellipsoids at $30 \%$ probability level, $\mathrm{H}$ atoms given arbitrary radii. atoms anisotropically for a total of 156 variables. $R=0.030, w R=0.044, S=1.36$, where non-Poisson $w^{-2}=\sigma^{2}\left(F^{2}\right)=\sigma(I)+0.0025 F^{2}$. Final $(\Delta / \sigma)_{\max }<0.01$, $\Delta \rho_{\max }=0.22(6)$ and $\Delta \rho_{\min }=-0.33(6) \mathrm{e} \AA^{-3}$ on final difference map. Atomic scattering factors and anomalous-dispersion corrections from International Tables for X-ray Crystallography (1974) and programs used those of Enraf-Nonius (1982) SDP.* Table 1 gives the atom parameters and Table 2 bond lengths and angles. Fig. 1 shows the molecule and numbering scheme.

Related literature. Structures of three other derivatives of 5-phenyl-1,3,2,4,6-dithiatriazine are in preparation or have been published: $\mathrm{PhCS}_{2} \mathrm{~N}_{3} \mathrm{Cl}_{2}$ (Graham, Cordes, Oakley \& Boeré, 1985), $\mathrm{PhCS}_{2} \mathrm{~N}_{3} \cdot \mathrm{C}_{7} \mathrm{H}_{8}$ (James, Craig, Cordes, Oakley \& Boeré, 1985), and $\left(\mathrm{PhCS}_{2} \mathrm{~N}_{3}\right)_{2}$ (Boeré, French, Oakley, Cordes, James, Craig \& Graham, 1985). The long $\mathrm{N}-\mathrm{S}$ bonds to the bridging NSN can be compared to the $1.692(8) \AA$ values observed in the related bicyclic molecule $\mathrm{F}_{2} \mathrm{~N}_{5} \mathrm{~S}_{3}$ (Weiss, Ruppert \& Appel, 1974).

We thank the National Science Foundation, the State of Arkansas, the Research Corporation, and the Natural Sciences and Engineering Research Council of Canada for financial support.

* Lists of structure factors and anisotropic temperature factors have been deposited with the British Library Lending Division as Supplementary Publication No. SUP 42418 (20 pp.). Copies may be obtained through The Executive Secretary. International Union of Crystallography, 5 Abbey Square, Chester CH1 2HU. England.

\section{References}

Boeré, R. T., French, C. L., OAkley, R. T., Cordes, A. W., James, J. A., Craig. S. L. \& Graham. J. B. (1985). In preparation.

Enraf-Nonius (1982). Structure Determination Package. EnrafNonius, Delft.

Graham, J. B., Cordes, A. W., OAkley, R. T. \& BoerÉ, R. T. (1985). Acta Crist. C41. 1835-1836.

International Tables for X-ray Crystallography (1974). Vol. IV. Birmingham: Kynoch Press. (Present distributor D. Reidel, Dordrecht.)

James, J. A., Craig, S. L., Cordes, A. W., Oakley, R. T. \& BOERE. R. T. (1985). In preparation.

JoHNSON, C. K. (1976). ORTEPII. Report ORNL-5138. Oak Ridge National Laboratory, Tennessee.

Main, P., Fiske, S. J., Hull, S. E., Lessinger, L., Germain, G., DEClERCQ, J.-P. \& WOOLFSON, M. M. (1982). MULTAN11/82. A System of Computer Programs for the Automatic Solution of Cry'stal Structures from X-ray' Diffraction Data. Univs. of York, England, and Louvain, Belgium.

Weiss, J., Ruppert, I. \& APPEL, R. (1974). Z. Anorg. Allg. Chem. 406, 329-336. 